\title{
B7-1, B7-2 and class II MHC molecules in idiopathic pulmonary fibrosis and bronchiolitis obliterans-organizing pneumonia
}

\author{
Y. Kaneko, K. Kuwano, R. Kunitake, M. Kawasaki, N. Hagimoto, N. Hara
}

\begin{abstract}
B7-1, B7-2 and class II MHC molecules in idiopathic pulmonary fibrosis and bronchiolitis obliterans-organizing pneumonia. Y. Kaneko, K. Kuwano, R. Kunitake, M. Kawasaki, N. Hagimoto, N. Hara. (C) ERS Journals Ltd 2000.

ABSTRACT: Interstitial lung diseases are thought to be associated with the infiltration of activated T-lymphocytes. To induce an effective immune response, antigen-presenting cells have to not only present antigenic peptide with major histocompatibility complex (MHC) molecules to T-lymphocytes but also express B7 molecules.

Therefore, the expression of B7-1, B7-2 and class II MHC molecules was investigated in lung tissues from patients with idiopathic pulmonary fibrosis (IPF) and bronchiolitis obliterans-organizing pneumonia (BOOP), and in normal lung parenchyma as a control, using immunohistochemical localization.

B7-1 and B7-2 were aberrantly expressed in bronchiolar and alveolar epithelial cells, and class II MHC molecules were also aberrantly expressed in bronchiolar epithelial cells in IPF. B7-1 was aberrantly expressed in bronchiolar epithelial cells in BOOP. There was no significant difference in the expression of these proteins in alveolar macrophages between IPF and control subjects. However, B7-2 and class II MHC molecule expression in alveolar macrophages was decreased in BOOP compared with that in control subjects. Expression of CD28 and CTLA-4, receptors for B7 molecules, was detected in infiltrating lymphocytes in lung tissues in IPF and BOOP.

It was concluded that bronchiolar and alveolar epithelial cells may actively participate in the pathophysiology of idiopathic pulmonary fibrosis through the aberrant expression of B7 and class II major histocompatibility complex molecules. The dysregulation of these molecules in epithelial cells may lead to the activation of autoreactive T-lymphocytes, which might contribute to the pathogenesis of fibrosing lung diseases.
\end{abstract}

Eur Respir J 2000; 15: 49-55.

Beside the specific signal delivered by an antigenic peptide in association with a major histocompatibility complex (MHC) molecule, T-cell activation requires a costimulatory signal that is provided by $\mathrm{B} 7-1$ and $\mathrm{B} 7-2$ molecules, which interact with the CD28 on T-cells $[1,2]$. All CD4+ and most CD8+ T-cells express CD28 constitutively, and CD28-deficient T-cells show a markedly reduced response to antigen stimulation [3]. A second ligand for B7 molecules is CTLA-4, which is expressed in T-cells after activation and may also regulate T-cell function [4]. Blocking of the $\mathrm{B} 7 / \mathrm{CD} 28$ pathway in vitro results in T-cell anergy [5,6], whereas in vivo blocking of the B7/CD28 pathway results in immunosuppression in animal models [7, 8].

Antigen-presenting cells (APCs) play an important role in regulating immune responses. One of the characteristic hallmarks of "professional" APCs is their expression of class II MHC molecules [9], which allows APCs to bind and present antigens to CD4+ T-cells. "Professional" APCs include bone marrow-derived B-cells, macrophages and dendritic cells [10,11].

Previous reports have suggested that epithelial cells in mucosal tissues lack B7 costimulatory molecules [12, 13].
Research Institute for Diseases of the Chest, Faculty of Medicine, Kyushu University, Fukuoka, Japan.

Correspondence: K. Kuwano, Research Institute for Diseases of the Chest, Faculty of Medicine, Kyushu University, 3-1-1, Maidashi, Higashiku, Fuknoka 812, Japan, Fax: 81926425389
Keywords: Autoimmunity
B7-1
B7-2
bronchiolitis obliterans organizing pneu- monia
class II major histocompatibility complex molecule
idiopathic pulmonary fibrosis

Received: January 111999

Accepted after revision October 151999

This work was supported by a Grant-inAid for Scientific Research (09670620) from the Ministry of Education, Science and Culture of Japan.
The absence of B7 costimulatory molecules has previously been shown to restrict the ability of epithelial cells to activate CD4+ T-cells [12]. However, recent studies have suggested that some "nonprofessional" APCs, such as keratinocytes [14], microglia [15] and gastric epithelial cells [16] also express B7 molecules and can lead to costimulation. Costimulating molecules participate in the pathogenesis of autoimmune diseases, the rejection of grafted organs and graft-versus-host disease. Antagonists of these molecules can prevent or ameliorate these conditions [17].

There is much clinical, pathological, and experimental evidence that some abnormality of immune function is involved in idiopathic pulmonary fibrosis (IPF), and patients with IPF often present clinical and serological evidence of autoimmunity. The pathogenesis of bronchiolitis obliterans-organizing pneumonia (BOOP), in which mononuclear cell infiltration into lung tissues is also found is also unknown. Since it is important to evaluate the expression of costimulating molecules in the activation of T-cells and the participation of autoimmunity in the pathogenesis of interstitial lung diseases, the expression of B7-1, B7-2 and class II MHC molecules in IPF and BOOP was investigated. 


\section{Materials and methods}

\section{Patients and control subjects}

This study of IPF was performed on lung samples obtained by means of open lung biopsy from 10 patients and by means of autopsy from four patients. The patient comprised 10 males and four females, whose ages ranged 4175 yrs (mean 59 yrs). Eleven were smokers and three were nonsmokers. The study of BOOP was performed on lung samples obtained by transbronchial biopsy from five patients. The patient comprised two males and three females, whose ages ranged 53-81 yrs (mean 62 yrs). Two were smokers and three nonsmokers. The diagnosis of IPF and BOOP was established on the basis of a combination of medical history, physical examination, laboratory tests, chest radiography, pulmonary function tests and the results from pulmonary biopsy specimens, according to previously described criteria [18-20]. In BOOP cases, at least three specimens were obtained via transbronchial biopsy. One case showed histological findings of bronchiolitis obliterans-organizing pneumonia, and four of those had organizing pneumonia with mononuclear cell infiltration into the interstitium. In all cases of BOOP, bronchoalveolar lavage (BAL) fluid cytological results and the phenotypic profile were consistent with a typical BAL fluid profile of BOOP. All cases of BOOP responded dramatically to corticosteroid treatment. The control subjects comprised ten patients with lung cancer consisting of a solitary pulmonary nodule, and were retrospectively free of any inflammatory disease, and demonstrated an absence of signs of restrictive or obstructive lung disease in the pulmonary function test. The controls comprised five males and five females, whose ages ranged 55-79 yrs (mean 65 yrs). Six were smokers and four nonsmokers. In all patients, a current infection with bacteria, mycobacteria or fungi was excluded by means of negative cultures of sputum or BAL fluid.

\section{Tissue preparation}

The tissue samples were fixed in $10 \%$ formalin overnight, and then embedded in paraffin. Two $5 \mu \mathrm{m}$ paraffin sections were adhered to slides pretreated with poly-L-lysine. These sections were dewaxed by washing three times for $5 \mathrm{~min}$ each in xylene, then rehydrated in 100\%, 95\% and $80 \%$ ethanol, for 5 min each, and finally rinsed with distilled water.

\section{Immunohistochemical localization of B7 and major his- tocompatibility complex molecules}

Hydrated autoclaving was used as a pretreatment before immunostaining, as previously described by SHIN et al. [21]. Following deparaffinization in xylene and rehydration in ethanol, the tissue sections were autoclaved for 20 min at $121^{\circ} \mathrm{C}$ in a glass pot filled with distilled water to completely immerse the sections and washed three times in $0.1 \mathrm{M}$ phosphate-buffered saline (PBS). Immunohistochemical localization was performed via a modified streptavidin/biotinylated peroxidase technique using a Histofine SAB-PO kit (Nichirei Corporation, Tokyo, Japan). Nonspecific protein staining was blocked with rabbit serum for $30 \mathrm{~min}$ at room temperature. The sections were incubated with mouse antihuman B7-1 (Immunotech, Marseille, France), antihuman B7-2 (PharMingen, San Diego, CA, USA) and antihuman MHC Class II antigen monoclonal antibody (PharMingen), which reacts with all major histocompatibility class II HLA-DR and $\mathrm{DP}$, and most DQ, antigens, overnight at $4{ }^{\circ} \mathrm{C}$. The sections were rinsed with PBS and incubated with biotinylated antimouse immunoglobulin $\mathrm{G}$ (IgG) (Nichirei Corporation) for $30 \mathrm{~min}$, and washed and treated with $0.3 \%$ hydrogen peroxide, to inhibit the activity of any endogenous peroxidase, in methanol for $30 \mathrm{~min}$. The slides were washed, incubated with streptavidin/ biotin/ peroxidase complex (Nichirei Corporation) for $30 \mathrm{~min}$ and developed according to the manufacturer's directions. For control incubations, specific antibodies were replaced with nonimmune mouse IgG (Santa Cruz, Santa Cruz, CA, USA). The intensity of staining was analysed independently by two observers used to immunohistochemical localization. The degree of staining was assessed semiquantitatively in each type of cell: grade $2,>50 \%$ of cells positive; grade $1,>10 \%$ of cells positive; and grade $0,<10 \%$ of cells positive. The criteria for distinguishing between alveolar and bronchiolar epithelium were as follows. When there was damage to type I pneumocytes, as evidenced by their widespread replacement by hyperplastic or atypical cells, but alveolar architecture was maintained, alveolar wall-lining cells were defined as type II cells. When there was destruction of the normal acinar architecture with considerable replacement fibrosis, such as honeycombing, cells lining the remodelling airspace were defined as bronchiolar epithelium.

\section{Immunohistochemical localization of CD28 and CTLA-4}

In order to detect lymphocytes expressing receptors enabling them to bind with sufficient avidity to costimulating molecules, immunohistochemical localization of CD28 and CTLA-4 was performed. This was carried out as described above without the autoclaving pretreatment, using goat polyclonal antihuman CD28 antibody (Santa Cruz) and antihuman CTLA-4 antibody (Santa Cruz). For control incubations. specific antibodies were replaced by goat serum (Nichirei, Corporation).

\section{Statistical analysis}

To determine statistical significance, the immunostaining grades were compared using the Mann-Whitney U-test and the intra- and interobserver correlations were analysed using Spearman's rank correlation coefficient (Abacus Concepts Statview J4.11 package). A p-value of $<0.05$ was considered significant.

\section{Results}

\section{Intra- and interobserver correlations}

Table 1 shows that intra- and interobserver correlations were good. The composite mean of two observers' grades was calculated and assessed as the grade of one patient when the difference between IPF or BOOP and controls was assessed. 
Table 1. - Intra- and interobserver correlation coefficients of the immunoreactivity grade

Observer Bronchiolar Alveolar Macrophages epithelium epithelium

\begin{tabular}{lllll}
\hline $\begin{array}{l}\text { Intraobserver } \\
\text { correlations }\end{array}$ & & & & \\
B7-1 & 1 & $0.986^{+}$ & $0.938^{+}$ & $0.732^{+}$ \\
& 2 & $0.924^{+}$ & $0.903^{+}$ & $0.779^{+}$ \\
B7-2 & 1 & $0.863^{+}$ & $0.907^{+}$ & $0.821^{+}$ \\
& 2 & $0.969^{+}$ & $0.936^{+}$ & $0.896^{+}$ \\
MHC class II & 1 & $0.967^{+}$ & $0.834^{+}$ & $0.828^{+}$ \\
& 2 & $0.916^{+}$ & $1.000^{+}$ & $0.940^{+}$ \\
Interobserver & & & & \\
correlations & & & & \\
B7-1 & - & $0.881^{+}$ & $0.576^{* *}$ & $0.729^{+}$ \\
B7-2 & - & $0.714^{+}$ & $0.834^{+}$ & $0.867^{+}$ \\
MHC class II & - & $0.827^{+}$ & $0.736^{+}$ & $0.861^{+}$ \\
\hline
\end{tabular}

$* *: \mathrm{p}<0.01 ;{ }^{+}: \mathrm{p}<0.0001$.

\section{Immunohistochemical localization of B7-1}

Figure 1 shows representative results of immunohistochemical localization of B7-1. Positive B7-1 signals were found in the cytoplasm of bronchiolar and alveolar epithelial cells, as well as alveolar macrophages in lung tissues from patients with IPF (fig. 1A and B). In normal lung parenchyma, positive signals were found in macrophages but not in epithelial cells (fig. 1C).

As shown in figure 2, the degree of B7-1 expression in bronchiolar and alveolar epithelial cells was significantly upregulated in IPF and BOOP compared with controls. There was no significant difference in B7-1 expression in macrophages between IPF and the controls, whereas there was a significant increase in BOOP compared with controls.

\section{Immunohistochemical localization of B7-2}

Figure 3 shows representative results of immunohistochemical localization of B7-2. B7-2 immunoreactivity was detected in bronchiolar and alveolar epithelial cells as well as alveolar macrophages in lung tissues from patients with IPF (fig. 3A and B). There were positive signals in alveolar macrophages in normal lung parenchyma (fig. 3C), but no positive signal in epithelial cells.

As shown in figure 2, the degree of B7-2 expression in bronchiolar and alveolar epithelial cells was significantly upregulated in IPF compared with controls. B7-2 expression in macrophages was significantly decreased in BOOP compared with the controls, whereas that in IPF was almost equal to that of the controls.

Immunohistochemical localization of class II major histocompatibility complex molecules

Figure 4 shows representative results of immunohistochemical localization of class II MHC molecules. Class II MHC molecule immunoreactivity was detected in bronchiolar epithelial cells, as well as alveolar macrophages in lung tissues from patients with IPF (fig. 4A and B). There were positive signals in alveolar macrophages in normal lung parenchyma (fig. 4C), but no positive signal in epithelial cells.
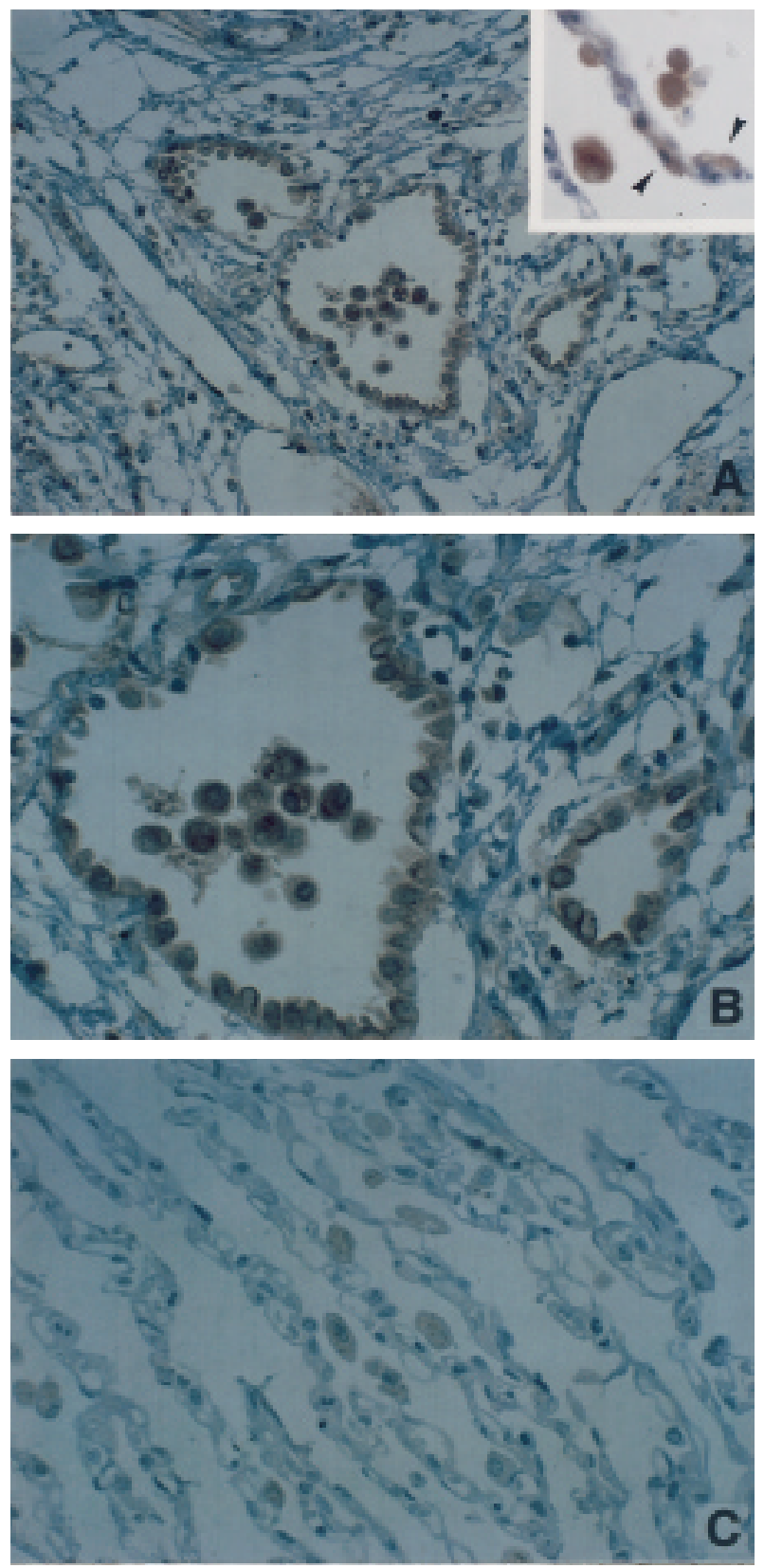

Fig. 1. - Immunohistochemical localization of B7-1. Positive signals were seen in: A, B) bronchiolar and alveolar epithelial cells (arrowheads in inset) and macrophages in idiopathic pulmonary fibrosis; and C) macrophages but not epithelial cells in normal lung parenchyma.

As shown in figure 2 the degree of class II MHC molecule expression in bronchiolar epithelial cells was significantly upregulated in IPF compared with controls. That on alveolar epithelial cells was not upregulated in IPF or BOOP. There was no significant difference in class II MHC molecule expression in macrophages between IPF and the controls, whereas that in BOOP was significantly decreased compared with that of the controls.

Immunohistochemical localization of CD28 and CTLA-4

Figure 5 shows representative results of immunohistochemical localization of CD28 and CTLA-4. CD28 and 


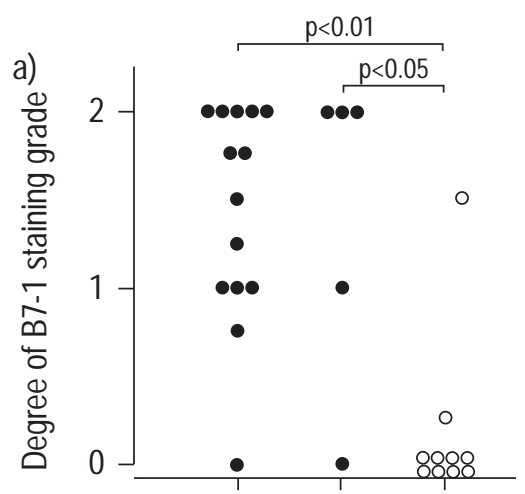

b)

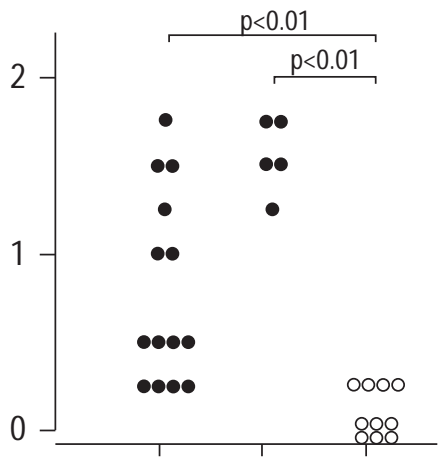

e)
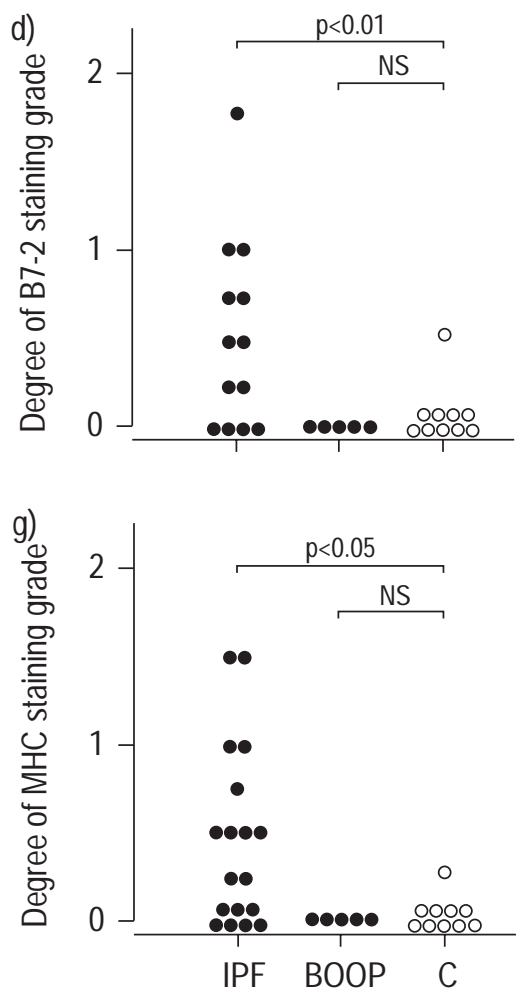

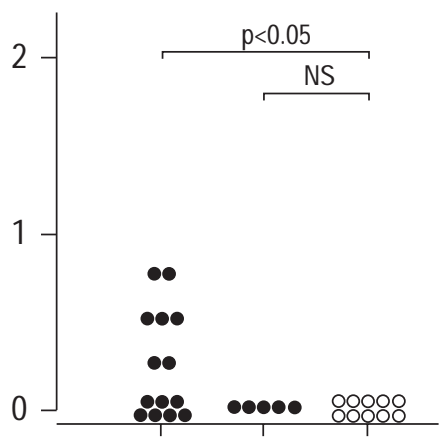

h)

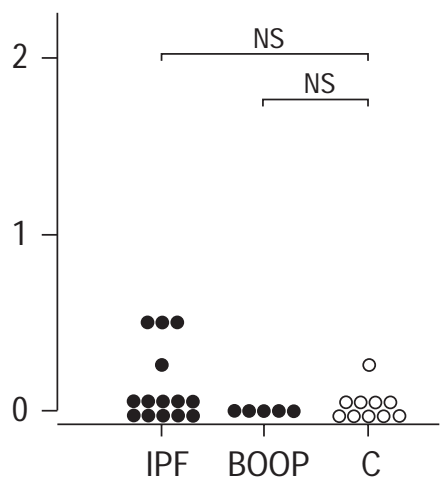

c)

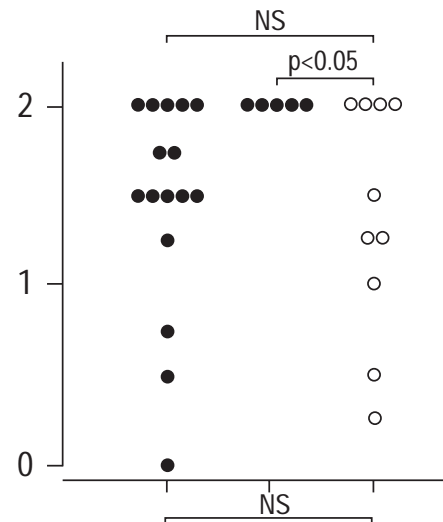

f)

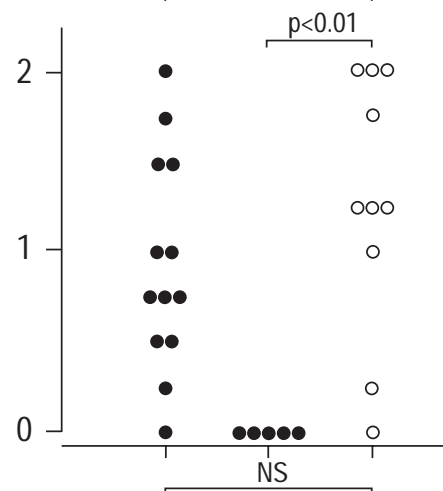

i)

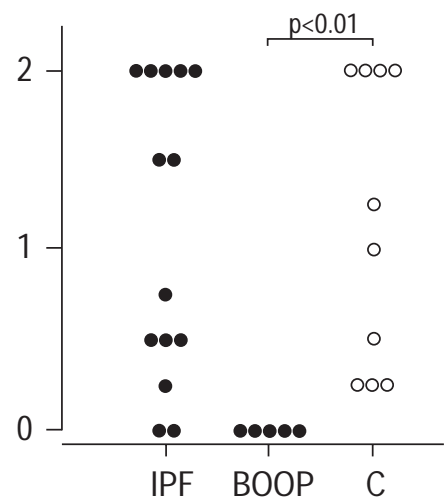

Fig. 2. - Quantification of immunostaining of: a-c) B7-1; d-f) B7-2; and g-i) class II major histocompatibility complex molecules on: a, d, g) bronchiolar and $\mathrm{b}, \mathrm{e}, \mathrm{h}$ ) alveolar epithelial cells; and $\mathrm{c}, \mathrm{f}, \mathrm{i}$ ) macrophages in idiopathic pulmonary fibrosis (IPF), bronchiolitis obliterans-organizing pneumonia (BOOP) and controls (C). Each point represents one individual. The immunostaining grade is as described in the Materials and Methods.

CTLA-4 immunoreactivity was detected in infiltrating lymphocytes in lung tissues from patients with IPF (fig. $5 \mathrm{~A}$ and $\mathrm{B}$, respectively) and BOOP. There were few positive signals on lymphocytes in normal lung parenchyma.

\section{Discussion}

It was observed that the costimulatory molecules B7-1, B7-2 and class II MHC molecules were aberrantly coexpressed in bronchiolar epithelial cells, and B7 molecules without class II MHC molecules were also expressed in alveolar epithelial cells in IPF. The B7 and class II MHC molecule expression in alveolar macrophages in IPF was not different from that of controls. In BOOP, B7-1 was aberrantly expressed in bronchiolar and alveolar epithelial cells, whereas B7-2 and class II MHC molecules were not detected in epithelial cells. In addition, B7-1 expression in macrophages in BOOP was significantly increased, whereas the expression of B7-2 and class II MHC molecules was significantly decreased in BOOP compared with that in controls. These findings suggest that the aberrant expression of B7-1, B7-2 and class II MHC molecules in epithelial cells in IPF and aberrant B7-1 expression in epithelial cells in BOOP may associate with the activation of immunological mechanisms.

Studies performed in transgenic mice support the possibility that ectopic expression of class I or II MHC molecules on "nonprofessional" APCs, such as islets of Langerhans cells, is not enough to activate T-cells [22]. However, coexpression of the appropriate costimulatory molecules, such as B7-1, in the islets activates these cells and results in autoimmune destruction of islets [23]. The 

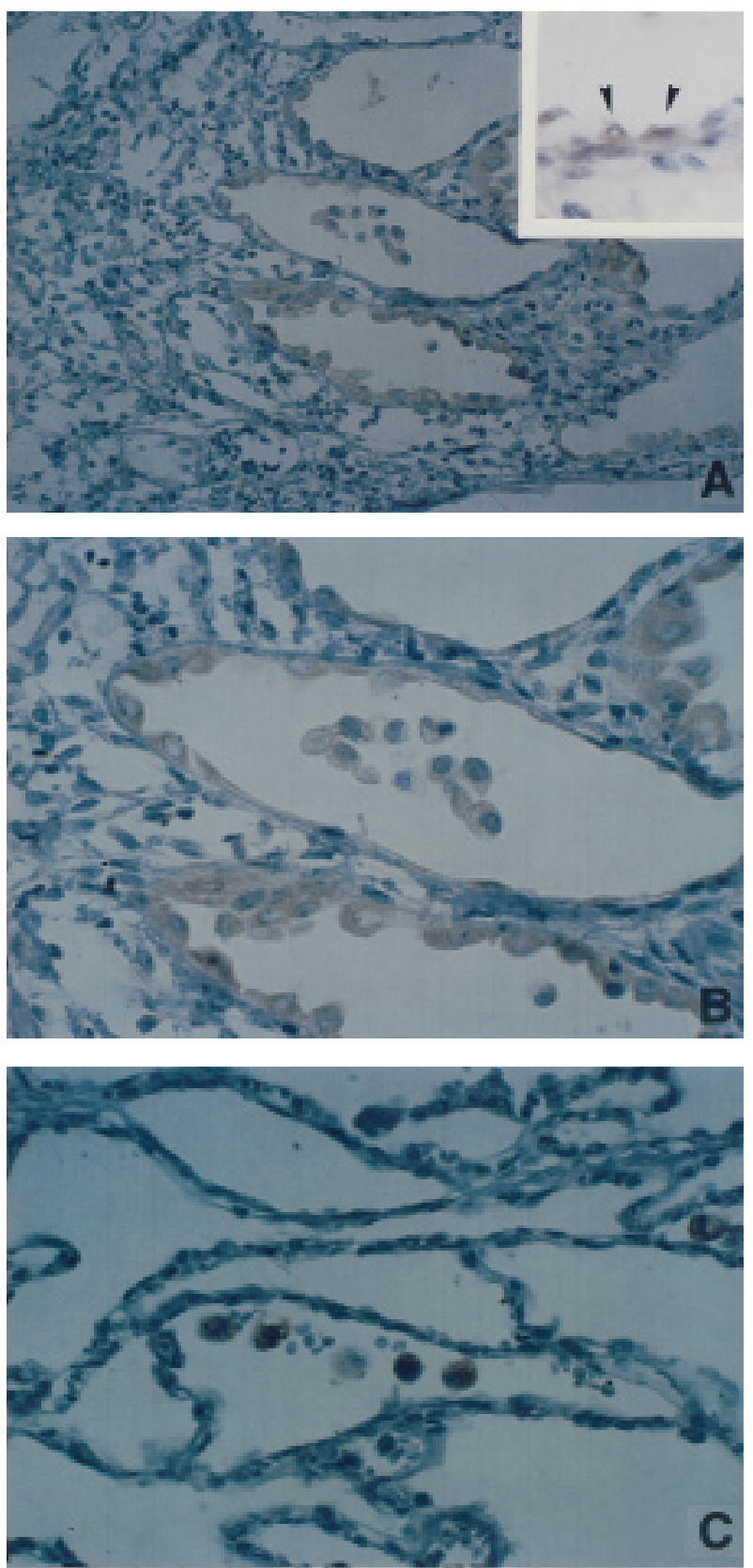

Fig. 3. - Immunohistochemical localization of B7-2. Positive signals were seen in: A, B) bronchiolar and alveolar epithelial cells (arrowheads in inset) and macrophages in idiopathic pulmonary fibrosis; and c) macrophages but not epithelial cells in normal lung parenchyma.

coexpression of B7 and class II MHC molecules in bronchiolar epithelial cells in IPF could be sufficient for the activation of T-cells and may lead to the autoimmune destruction of these cells. As well as in IPF, there was aberrant expression of B7-1 in bronchiolar epithelial cells in BOOP, while positive signals for B7-2 and class II MHC molecules were not present. It has been demonstrated that expression of B7-1 alone does not lead to the removal of tolerance to autoantigens in B7-1 transgenic mice $[24,25]$. Therefore, B7-1 expression in bronchiolar epithelial cells in BOOP may be of no significance for the activation of autoreactive CD4+ T-cells.
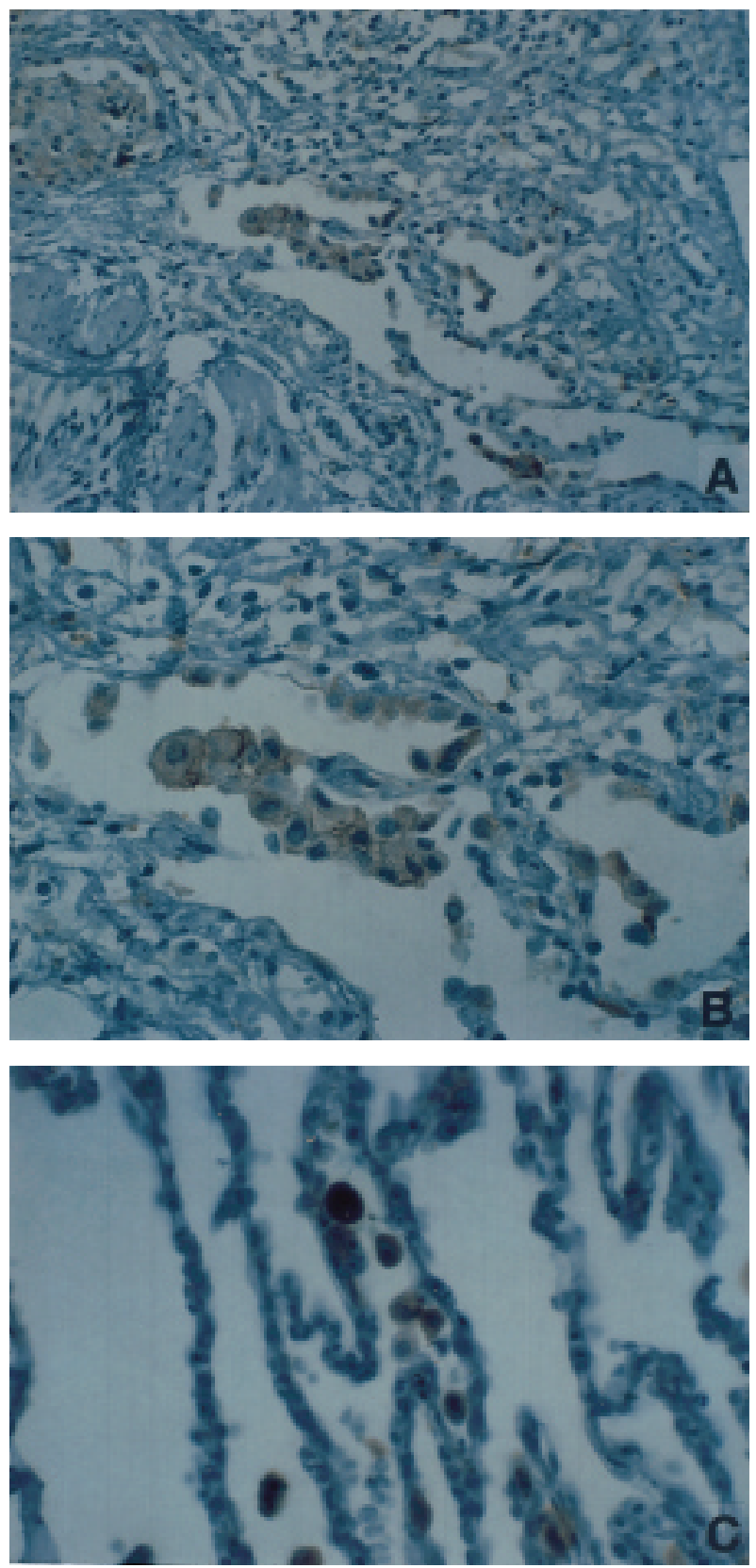

Fig. 4. - Immunohistochemical localization of class II major histochemical complex molecules. Positive signals were seen in: A, B) bronchiolar and alveolar epithelial cells and macrophages in idiopathic pulmonary fibrosis; and C) macrophages but not epithelial cells in normal lung parenchyma.

The outcome of an immune response involves a balance between CD28-mediated T-cell activation and CTLA-4mediated downregulation. In this study, CD28 and CTLA4 were expressed in infiltrating lymphocytes surrounding bronchiolar and alveolar epithelial cells in IPF. It has previously been demonstrated that almost equal numbers of CD4+ and CD8+ T-lymphocytes infiltrate the lung tissue of patients with IPF [26]. These lymphocytes express Fas ligand (FasL), which is one of the major cytotoxic effectors of T-cells and natural killer cells, and indicates the activation of T-cells [26]. It has also been demonstrated 

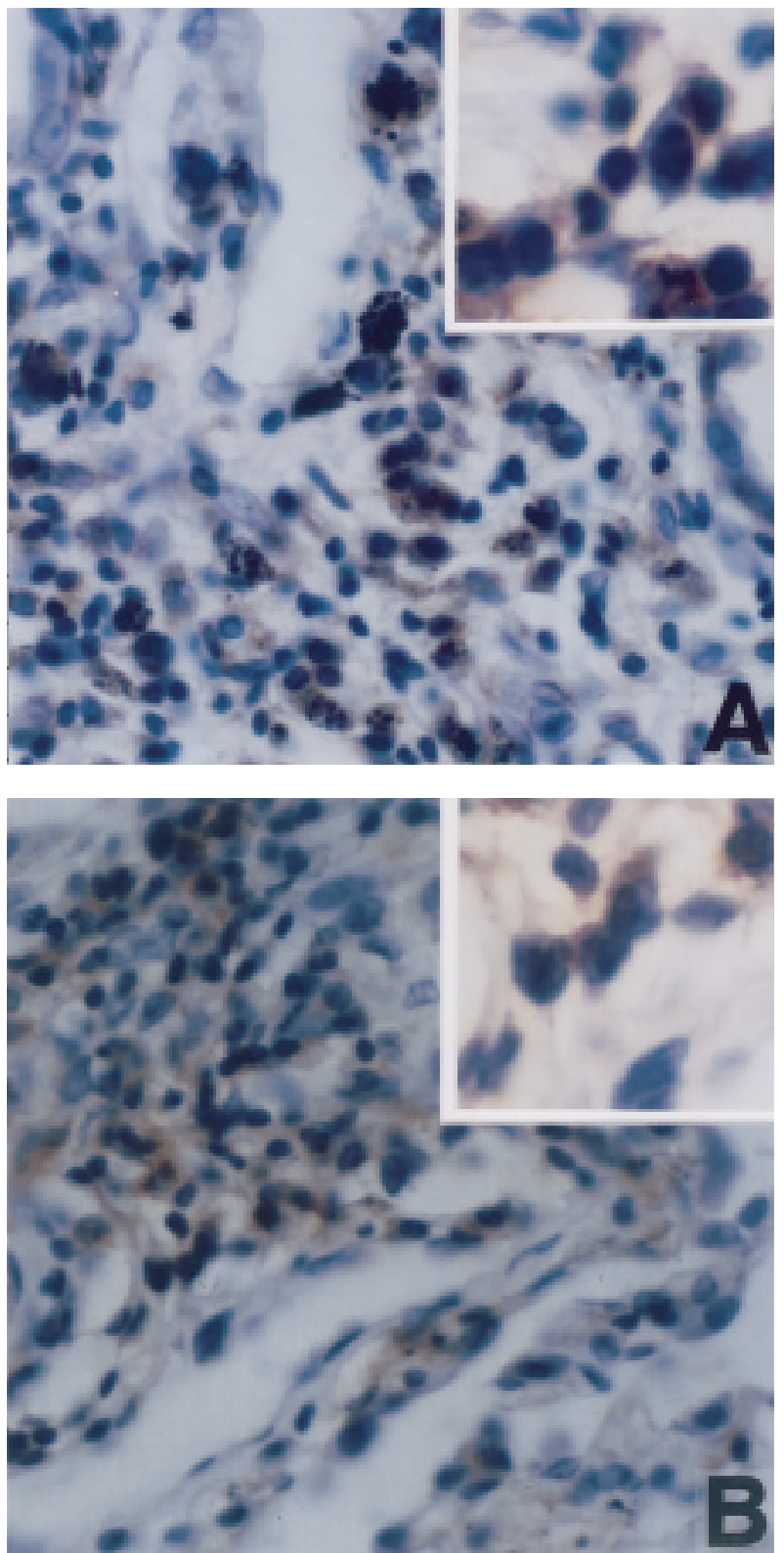

Fig. 5. - Immunohistochemical localization of CD28 and CTLA-4 Positive signals for: A) CD28; and B) CTLA-4 were seen in infiltrating lymphocytes in idiopathic pulmonary fibrosis.

that there was deoxyribonucleic acid damage and apoptosis in bronchiolar and alveolar epithelial cells in IPF [27]. These results suggest that autoreactive T-cells may be induced by the association of CD28 with the aberrant expression of B7 and MHC molecules in lung epithelial cells. These epithelial cells may be attacked by lymphocytes through cytotoxic effectors such as FasL and perforin, resulting in the loss of normal lung parenchyma and pulmonary fibrosis.

Recently, Sigal et al. [28] demonstrated that a combination of both anti-B7-1 and -B7-2 monoclonal antibody blocked cytotoxic T-lymphocytes (CTL) responses using MHC class II-deficient mice lacking most CD4+ cells, indicating that the T-helper cell-independent activation of CTLs is dependent upon the B7-costimulatory signals supplied to the CD8+ T-cells. Furthermore, it was demonstrated that transfecting poorly immunogenic class II MHC-negative tumour cells with B7-1 increases the ability of mice to reject such cells [29]. Therefore, the aberrant expression of B7 in lung epithelial cells without class II MHC molecules may also have the ability to activate autoimmunity in CD8+ T-cells.

Alveolar macrophages have been shown to be defective in activating T-cells and initiating inflammatory responses $[30,31]$. CHELEN et al. [32] demonstrated that alveolar macrophages were not able to present antigens effectively to T-cells due to the insufficient expression of B7 molecules [32]. B7-1, B7-2 and class II MHC molecules in alveolar macrophages in IPF were expressed as in controls, whereas B7-2 and class II MHC expression were suppressed in BOOP. If alveolar macrophages play a major role in downregulating immune responses in the lung and in immunological tolerance to inhaled antigens, the downregulation of B7-2 and class II MHC molecules may be the mechanism suppressing the immunological reaction in BOOP. Since the sizes of specimens were small, the number of bronchioles assessed in lung tissues from patients with BOOP was smaller than that in IPF or controls. The number of cases of BOOP we examined was also small; further study is required to address this speculation.

In summary, the aberrant coexpression of B7 and class II major histocompatibility complex molecules in bronchiolar epithelial cells, and that of B7 molecules in alveolar epithelial cells in idiopathic pulmonary fibrosis, were first demonstrated. In bronchiolitis obliterans-organizing pneumonia, B7-1 was aberrantly expressed in lung epithelial cells, whereas B7-2 and class II major histocompatibility complex molecules were suppressed in macrophages. CD28 and CTLA4 immunoreactivity was detected in lymphocytes that had infiltrated lung tissues from patients with idiopathic pulmonary fibrosis and bronchiolitis obliteransorganizing pneumonia. In view of the known properties of B7 and class II major histocompatibility complex molecules, the aberrant expression and upregulation of these molecules may activate T-cells to react against self antigens on epithelial cells, which may lead to epithelial cell damage and pulmonary fibrosis.

\section{References}

1. Schwartz RH. A cell culture model for T lymphocyte clonal energy. Science 1990; 248: 1349-1356.

2. Linsley PS, Ledbetter JA. The role of the CD28 receptor during T cell responses to antigen. Annu Rev Immunol 1993; 11: 191-212.

3. Green JM, Noel PJ, Sperling AI, et al. Absence of B7dependent responses in CD28-deficient mice. Immunity 1994; 1: 501-508.

4. Brunet JF, Denizot F, Luciani MF, et al. A new member of immunoglobulin superfamily-CTLA-4. Nature 1987; 328: 267-270.

5. Tan P, Anasetti C, Hansen JA, et al. Induction of alloantigen-specific hyporesponsiveness in human $\mathrm{T}$ lymphocytes by blocking interaction of CD28 with its natural ligand B7/BB1. J Exp Med 1993; 177: 165-173.

6. Gimmi CD, Freeman GJ, Gribben JG, Gray G, Nadler LM. Human T-cell clonal anergy is induced by antigen 
presentation in the absence of B7 costimulation. Proc Natl Acad Sci USA 1993; 90: 6586-6590.

7. Lenschow DJ, Zeng Y, Thistlethwaite JR, et al. Longterm survival of xenogeneic pancreatic islet grafts induced by CTLA-4 Ig. Science 1992; 257: 789-792.

8. Linsley PS, Wallace PM, Johnson J, et al. Immunosuppression in vivo by a soluble form of the CTLA-4 T cell activation molecule. Science 1992; 257: 792-795.

9. Unanue ER. Cellular studies on antigen presentation by class II MHC molecules. Curr Opin Immunol 1992; 4: 63-69.

10. Azuma M, Ito D, Yagita $\mathrm{H}$, et al. B70 antigen is a second ligand for CTLA-4 and CD28. Nature 1993; 366: 76-79.

11. Hathcock KS, Laszlo G, Dickler HB, Bradshaw J, Linsley PS, Hodes RJ. Identification of an alternative CTLA-4 ligand costimulatory for T cell activation. Science 1993; 262: 905-907.

12. Hagerty DT, Evavold BD, Allen PM. Regulation of the costimulator B7, not class II major histocompatibility complex, restricts the ability of murine kidney tubule cells to stimulate CD4+ T cells. J Clin Invest 1994; 93: 1208 1215.

13. Bloom S, Simmonds D, Jewell DP. Adhesion molecules intercellular molecule 1 (ICAM-1), ICAM-3 and B7 are not expressed by epithelium in normal or inflamed colon. Clin Exp Immunol 1995; 101: 157-163.

14. Simon JC, Dietrich A, Mielke V, et al. Expression of the B7/BB1 activation antigen and its ligand CD28 in T cellmediated skin diseases. J Invest Dermatol 1994; 103: 539-543.

15. Williams K, Ulvestad E, Antel JP. B7/BB-1 antigen expression on adult human microglia studied in vitro and in situ. Eur J Immunol 1994; 24: 3031-3037.

16. Gang Y, Barrera C, Xuejun F, et al. Expression of B7-1 and $\mathrm{B} 7-2$ costimulatory molecules by human gastric epithelial cells. Potential role in CD4+ T cell activation during Helicobactor pylori infection. J Clin Invest 1997; 99: $1628-1636$.

17. Bluestome JA. New perspectives of CD28-B7-mediated $\mathrm{T}$ cell costimulation. Immunity 1994; 2: 555-559.

18. Crystal RG, Bitterman PB, Rennard SI, Hance AJ, Keogh BA. Interstitial lung diseases of unknown cause. Disorders characterized by chronic inflammation of the lower respiratory tract (first of two parts). N Engl J Med 1984; 310: 154-166.

19. Crystal RG, Fulmer JD, Roberts WC, Moss ML, Line BR, Reynolds HY. Idiopathic pulmonary fibrosis. Clinical, histologic, radiographic, physiologic, scintigraphic, cytologic, and biochemical aspects. Ann Intern Med 1976; 85: 769-788.

20. Epler GM, Colby TV, McLoud TC, Carrington CB,
Gaensler EA. Bronchiolitis obliterans organizing pneumonia. N Engl J Med 1985; 312: 152-158.

21. Shin RW, Iwaki T, Kitamoto T, Tateishi J. Methods in laboratory investigation. Hydrated autoclave pretreatment enhances TAU. Immunoreactivity in formalin-fixed normal and Alzheimer's disease brain tissues. Lab Invest 1991; 64: 693-702.

22. Burkly LC, Lo D, Flavell RA. Tolerance in transgenic mice expressing major histocompatibility molecules extrathymically on pancreatic cells. Science 1990; 248: 1364-1368.

23. Guerder S, Meyerhoff J, Flavell RA. The role of the T cell costimulator B7-1 in autoimmunity and the induction and maintenance of tolerance to peripheral antigen. Immunity 1994; 1: 155-166.

24. Guerder S, Pivarella DE, Linsley PS, Flavell RA. Costimulator B7-1 confers antigen-presenting-cell function to parenchymal tissue and in conjunction with tumor necrosis factor a leads to autoimmunity in transgenic mice. Proc Natl Acad Sci USA 1994; 91: 5138-5142.

25. Harlan DM, Hengartner H, Huang ML, et al. Mice expressing both B7-1 and viral glycoprotein on pancreatic beta cells along with glycoprotein specific transgenic $\mathrm{T}$ cells develop diabetes due to a breakdown of T-lymphocyte unresponsiveness. Proc Natl Acad Sci USA 1994; 91: 3137-3141.

26. Kuwano K, Miyazaki H, Hagimoto N, et al. The involvement of Fas-Fas ligand pathway in fibrosing lung diseases. Am J Respir Cell Mol Biol 1999; 20: 53-60.

27. Kuwano K, Kunitake R, Kawasaki M, Nomoto Y, Hagi-

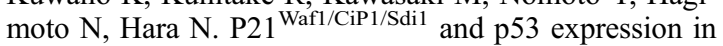
association with DNA strand breaks in idiopathic pulmonary fibrosis. Am J Respir Crit Care Med 1996; 154: 477-483.

28. Sigal LJ, Reiser H, Rock KL. The role of B7-1 and B7-2 costimulation for the generation of CTL responses in vivo. J Immunol 1998; 161: 2740-2745.

29. Townsend S, Allison J. Tumor rejection after direct costimulation of CD8 $+\mathrm{T}$ cells by B7-transfected melanoma cells. Science 1993; 259: 368-370.

30. Ettensohn DB, Lalor PA, Roberts NJ. Human alveolar macrophage regulation of lymphocytes proliferation. $\mathrm{Am}$ Rev Respir Dis 1986; 133: 1091-1096.

31. Lyons CR, Ball EJ, Toews GB, Weissler JC, Stastny P, Lipscomb MF. Inability of human alveolar macrophages to stimulate resting $\mathrm{T}$ cells correlates with decreased antigen-specific $\mathrm{T}$ cell-macrophage binding. J Immunol 1986; 137: 1173-1180.

32. Chelen CJ, Fang Y, Freeman GJ, et al. Human alveolar macrophages present antigen ineffectively due to defective expression of B7 costimulatory cell surface molecules. J Clin Invest 1995; 95: 1415-1421. 